\title{
Capacity of Shear Wave Elastography in Diffuse Liver Diseases in Children
}

\author{
M I Pykov ${ }^{1}$, N E Kuzmina ${ }^{2 *}$, N M Rostovtsev ${ }^{3}$ and A Yu Kinzersky ${ }^{4}$ \\ ${ }^{1}$ Division of Pediatric Radiology, Russian Medical Academy of Postgraduate Education, Moscow \\ ${ }^{2}$ Department of Ultrasound Diagnostics, Chelyabinsk Regional Children Clinical Hospital, Russia
}

${ }^{3}$ Department of Pediatric Surgery, Federal State Budgetary Institution of Higher Education (FSBEI HE) \& South Ural State Medical University (SUSMU), Russia

${ }^{4}$ Professor, Kinzersky Clinic, Chelyabinsk

Submission: March 26, 2019; Published: April 26, 2019

*Corresponding author: Natalya Kuzmina, Chelyabinsk Regional Children Clinical Hospital, Department of Ultrasound Diagnostics, 454087

Chelyabinsk, Blurher str, house 42 A, Russia

\begin{abstract}
Two hundred and thirty children aged 3 to 18 years were included in the study, among them 200 were practically healthy (control group) and 30 patients aged 2 to 17 years had chronic liver diseases (study group). All patients underwent standard liver tests and twodimensional shear wave elastography (Aixplorer device (Supersonic Imagine, France, convex sensor acting within the medium-frequency range of 1-6MHz). Ten measurements of Young modulus (Emean) were made in different segments of the right and left liver lobes with subsequent data averaging. In patients with chronic liver disease Young modulus values were significantly higher than in patients of the control group (Emean median was $7.05 \mathrm{kPa}$ and $5.00 \mathrm{kPa}$, interquartile range was 6.40-9-18 and 4.70-5.38kPa correspondingly) ( $\mathrm{p}<0.001$ )

Ultrasound elastometry can be successfully used in complex assessment of liver diseases in children for dynamic monitoring in the group of patients with chronic liver diseases. Young modulus value of $6.35 \mathrm{kPa}$ will enable to reveal children who need additional examination when healthy children undergo screening evaluation by means of $80 \%$ sensitivity and $100 \%$ specificity tests.
\end{abstract}

Keywords: Ultrasound elastography; Shear wave elastography; Stiffness; Young modulus; Chronic liver disease; Fibrosis; Liver; Children

\section{Introduction}

Chronic diffuse liver diseases are an urgent issue in present children gastroenterology. The interest to this group of liver diseases is due to their increasing incidence, frequent severe course, tendency to progression and unfavorable outcomes. This problem requires great attention as the course of the diseases is often insufficiently symptomatic because of the great compensatory capacities of the organ. Clinical manifestations and patient presentation often take place when severe morphological changes have occurred, and adaptation and compensatory mechanisms have already been wasted [1]. Regardless of the etiology, cirrhosis is the cause of fatal outcome in patients due to the development of complications, i.e. hemorrhage from the esophageal varices, ascites, encephalopathy, hemorrhagic syndrome, transformation to hepatocellular carcinoma.

Various infectious agents such as hepatitis B, C, D, G viruses, cytomegalovirus, Epstein-Barr virus as well as autoimmune liver diseases, cystic fibrosis, metabolism diseases, etc. may act as etiologic factors causing liver fibrosis and cirrhosis in children. There is a direct correlation between the intensity of cirrhotic changes in the liver and the degree of cytolytic enzymes activity in viral hepatitis in children. In children with liver cirrhosis developing due to the conditions not related to the viral hepatitis pathologic process activity may be contemplated as a factor contributing to the progression of liver affection to cirrhosis. Thus, cirrhotic changes which are observed in diseases with high activity (autoimmune hepatitis, biliary atresia) develop within a short period. While diseases occurring with normal activity (congenital hepatic fibrosis) and insignificantly increased activity (cystic fibrosis) progression to liver cirrhosis occurs much slower. Long term catamnesis observations of liver cirrhosis and chronic viral hepatitis course have shown that cirrhosis occurs sufficiently early from the moment of its identification and is not the outcome of chronic viral hepatitis [2]. In spite of the increased experience in the study of viral liver diseases, characteristics of clinical manifestations, diagnosis, treatment and prevention of viral hepatitis A, B, C, D, E as well as metabolic, hereditary liver diseases i.e., galactosemia, $\alpha$-1-antitripcin, Wilson-Konovalov disease, Alagil syndrome, Goshe disease, etc. still present some difficulties. They require 
technically and methodically complex approaches and committed pathogenetic therapy. Late diagnosis of many diseases results in unfavorable outcome so that there remains the only option i.e. orthotopic hepatic transplantation. It is practically impossible to extrapolate the study results obtained from adult patients because of the anatomico-physiological characteristics of a child because of his development rates, formation of all organs and systems [3].

Liver biopsy is still the basic diagnostic and predictive procedure in evaluation of liver diseases in children. Specific histological characteristics are important to diagnose hepatitis, cholestatic liver affections, steatosis, vascular conditions, storage diseases $[4,5]$.

Biopsy is of utmost importance in case of crossed syndromes or non-typical clinical manifestation when only histologic sample can help solving diagnostic dilemma and give a chance of timely adequate therapy. Indications to perform liver biopsy are numerous and they increase in number when the current knowledge of etiology, molecular basis and therapy options of liver diseases in children increase. Development of alternative diagnostic methods and improvement of liver visualization techniques change the role of biopsy. The biopsy procedure is often complicated by technical difficulties connected with anesthesia, availability of trained staff and equipment, smaller sizes of the organ and a smaller sample. It may cause such complications as pain syndrome, profuse hemorrhage, formation of subcapsular liver hematomas, development of biliary peritonitis, etc. [6]. Standard liver biopsy averages $1 / 50.000$ of the whole liver that is why the sampling error of 20 $30 \%$ is significant. It is necessary for the clinicians to cooperate with pathology doctors who have a clear picture of hepatobiliary problems in children. Diagnostic errors made by unexperienced specialists were registered in more than $25 \%$ of patients. Biliary atresia is a challenge for clinicians and pathology doctors because a missed opportunity of early diagnosis results in a missed opportunity of surgical correction. The feasibility of intra- and inter-observational study of biopsy samples is well known and can be the main factor limiting liver biopsy capacity [7].

That is why considering all difficulties of histologic verification specialists are motivated to search for noninvasive techniques which will enable not only to reveal changes in the liver but also to carry on dynamic observation of fibrosis process.

Among non-invasive techniques ultrasound elastography is of special interest. This method enables to evaluate tissue elastic properties while carrying on routine ultrasound exam by means of measuring the velocity of shear wave values $(\mathrm{m} / \mathrm{s})$ or Young modulus $(\mathrm{kPa})$ in the organs and tissues of interest. Changes in tissue elasticity are registered by various types of ultrasound elastometry, i.e. transient elastography, two-dimensional shear wave elastography, point shear wave elastography. Performing two-dimensional shear wave elastography quantitative information in the form of digital data of the shear wave velocity (or Young modulus) in the area of interest (light window) is obtained. Areas with different velocity values of shear wave (or various values of Young modulus) are mapped by different colors. It is the digital values of these parameters that determine the color in the area of interest [8].

Shear wave elastography can be successfully used in the complex evaluation of liver parenchyma when it is affected and differentiation of diffuse pathology in B-mode is problematic. This ultrasound approach is intensively studied, but not in pediatrics [9-12]. Diagnostic efficiency of two-dimensional shear wave elastography was proved by the studies performed in the adult population. As a result, the threshold elastometry values for each stage of fibrosis were established [13-15].

The purpose of our investigation has been to study the values of liver stiffness in children with liver chronic diseases and performing a routine ultrasound exam to establish the values of Young modulus which will enable to reveal patients with uncertain chronic liver conditions requiring additional complex examination.

\section{Material and Methods}

Two hundred and thirty children aged 3 to 17 years were included in the study. Among them there were 200 practically healthy children aged 3 to 18 years (control group) and 30 patients aged 2 to 17 years with chronic liver diseases (study group).

In the group of children with hepatitis the patients were distributed in the following way: cryptogenic hepatitis - 10, autoimmune hepatitis - 4, hepatitis of unspecified genesis 3 , liver cirrhosis - 3, hepatitis of cytomegaloviral etiology -2 , storage disease -2 , unspecified liver fibrosis, primary sclerosing cholangitis -2 , virus $\mathrm{C}$ hepatitis -1 , herpes-viral hepatitis -1 (Figure 1).

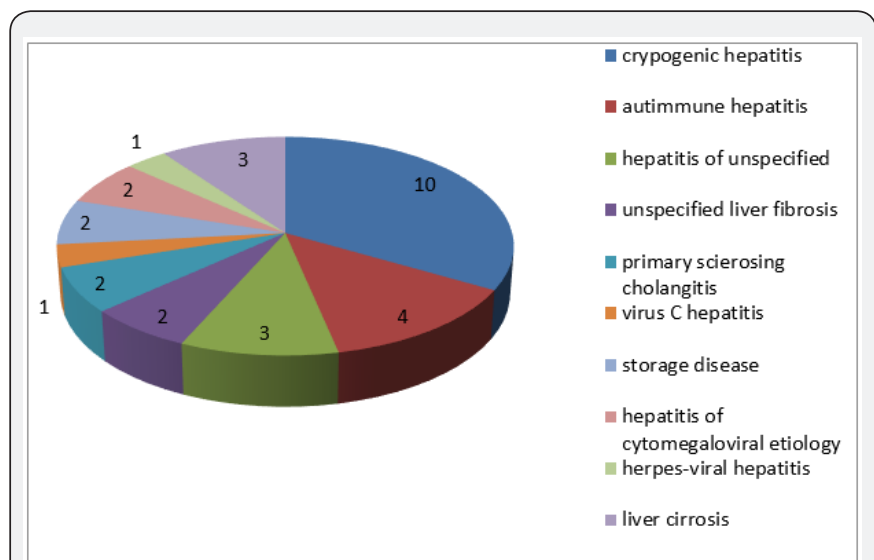

Figure 1: Distribution of patients in the group with hepatitis.

All children underwent standard ultrasound study of abdominal organs in the grey scale mode supplemented by two-dimensional shear wave elastography on Aixplorer device 
(Supersonic Imagine, France) by broadband convex sensor acting within frequency range of 1-6MHz. Elastometry was made when a patient was fasting, breathing normally, in elder children during breath-holding for not more than $10 \mathrm{sec}$, patients were in a supine position. There were subcostal, intercostal and epigastric accesses. The sensor was placed perpendicularly to the body surface with minimal pressure. Measurements were taken in the areas free from the vascular structures, fixing the zone of scanning at the depth of $3-5 \mathrm{~cm}$ from the capsule, in different segments of the right and left hepatic lobes, taking into account literature data testifying absence of significant differences between stiffness in the right and left lobe. The investigation was completed after getting 10 informative measurements with average values of Young modulus $(\mathrm{kPa})$ - Emean, at stabilization of the image to get a homogeneous coloring of the light window and color filling for more than $90 \%$. By the results of the measurements arithmetic mean value of Young modulus characterizing liver stiffness was calculated.

Informed consent was obtained from the legal representatives of all patients. The study was approved by the Ethics committee FGBOU DPO "Russian Medical Academy of Postgraduate Professional Education" of Health Ministry of the Russian Federation and local Ethics committee of GBUZ "Chelyabinsk Regional Children Clinical Hospital".

Statistical analysis of data was performed by IBM SPSS Statistics 19 pack. Most of the quantitative values did not follow normal distribution, that is why methods of nonparametric statistics were used. All quantitative values were presented as $\mathrm{M}$ (mean value), $\sigma$ (standard deviation), median ( $50^{\text {th }}$ percentile), $25^{\text {th }}-75^{\text {th }}$ percentiles of both minimal and maximal values. Comparison of quantitative parameters was performed using Mann-Whitney test, qualitative ones were compared by Fisher criterion of accuracy. Differences $(p>0.0) 5$ were considered significant.

\section{Results}

During the investigation of the study group of children hepatomegaly was revealed in 28 patients $(93,3 \%)$, splenomegaly in 17 (56.7\%), signs of portal hypertension were found in 7 children (23.3\%). Twenty-three children $(76.7 \%)$ were ill not more than 3 years, one child was ill up to 5 years $(3.3 \%)$, in the rest of the children ( 6 patients) the duration of the disease was more than 5 years (20\%). All the patients of the study group underwent examination in Chelyabinsk Regional Clinical Hospital. They were admitted to the hospital during the development of symptoms (abdominal, dyspeptic, asthenia -vegetative syndrome, complaints of malaise and fatigue, abdominal pain, vomiting, skin and sclera icterus combined with increased transaminase in the biochemical blood analysis), they were also examined for isolated increase of liver enzymes resulting in cholestasis and cytolysis syndrome. Histological verification was done in 18 patients of the study group. During this verification we faced the absence of unanimous consent on the pathomorphological evaluation of biopsy samples: evaluation of fibrosis stage according to different scales Knodell, Metavir, Desmet included just descriptive picture of the sample without any conclusion of fibrosis stage. Autoimmune hepatitis was found in 4 children based on presence of ANA- and SMAantibodies. Nine patients underwent CT and MRI, 3 patients had radioisotope imaging.

Elastography characteristics of changes in the liver in all patients with hepatitis showed the presence of heterogeneous elastometry picture: from homogenous coloring in dark blue and light blue tones and absence of areas of increased stiffness (i.e. qualitative characteristics (range of colors) were not practically different from the control group to yellow-orange and red coloring in the area of interest (Figure 2 \& 3).
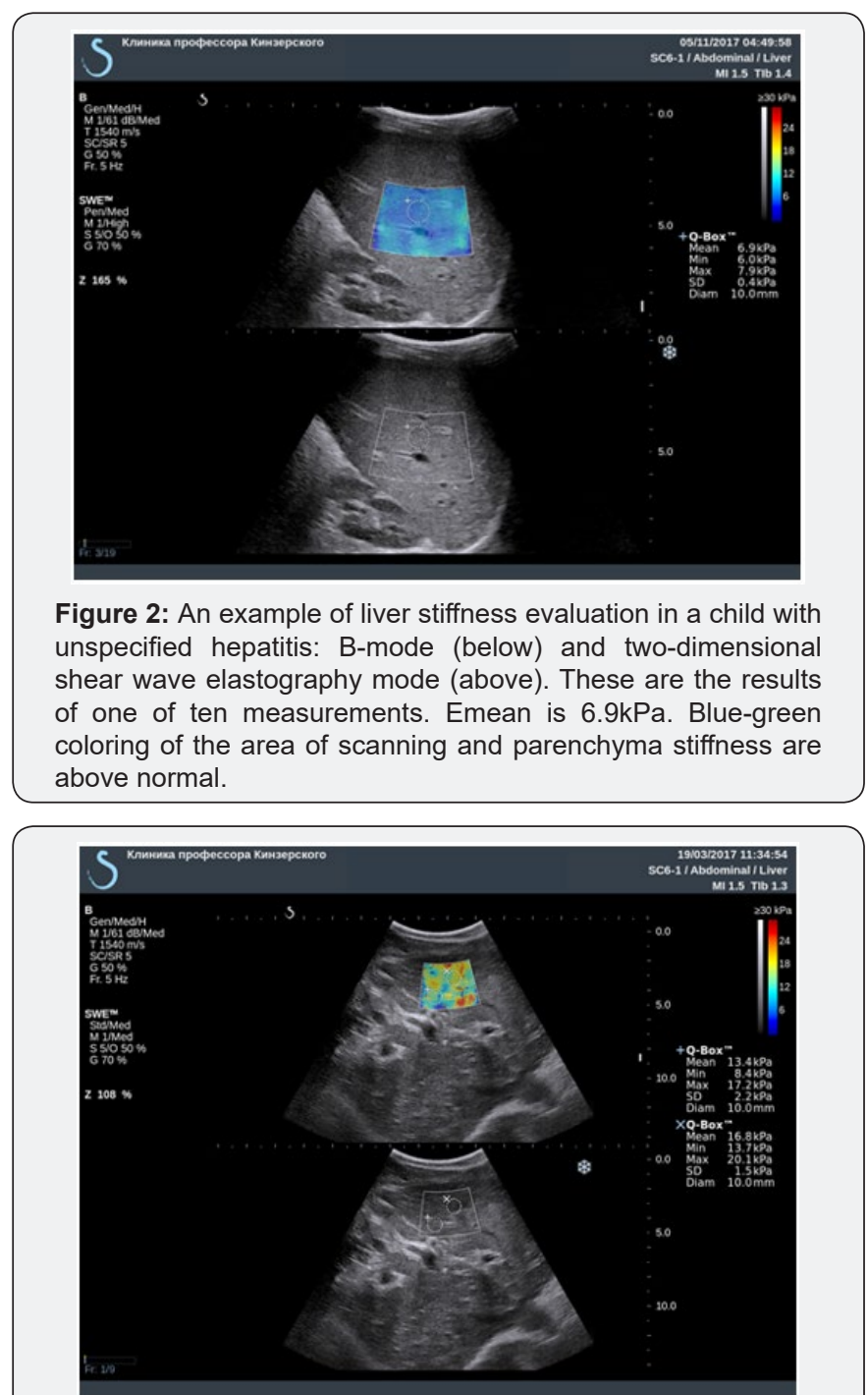

Figure 3: An example of liver stiffness evaluation in a child with chronic hepatitis: B-mode (below) and two-dimensional shear wave elastography mode (above). These are the results of one of ten measurements. Emean is $16.8 \mathrm{kPa}$. Heterogenous green-yellow with red areas coloring of the area of scanning corresponds to the changed liver stiffness (cirrhosis). 


\section{Juniper Online Journal of Case Studies}

Earlier we studied liver stiffness in 200 practically healthy children aged 3 to 18 years. Young modulus values were considered as standard [9]. Young modulus values in the study and control groups are presented Table 1. Comparing groups of patients with chronic liver diseases and those from the group of practically healthy children significant differences of Young modulus values were obtained (Emean) $(\mathrm{p}<0.000)$.

Table 1: Liver parenchyma stiffness (Emean, $\mathrm{kPa}$ ) in children of the control group and children with hepatitis

\begin{tabular}{|c|c|c|c|c|c|}
\hline Groups & $\mathbf{M} \pm \mathbf{m}$ & $\boldsymbol{\sigma}$ & Median & $\mathbf{2 5}^{\text {th }} \mathbf{7 5 ^ { \text { th } }}$ Percentile & Minimal-Maximal Values \\
\hline Control group ( $\mathrm{n}=200)$ & $5.01 \pm 0.03$ & 0.49 & 5 & $4.70-5.38$ & $3.00-6.30$ \\
\hline Group of children with hepatitis $(\mathrm{n}=30)$ & $7.89 \pm 0.43$ & 2.34 & 7.05 & $6.40-9.18$ & $4.80-14.40$ \\
\hline \multicolumn{7}{|c|}{ Notes: comparing two groups $\mathrm{p}<0.001$} \\
\hline
\end{tabular}

Considering insufficient number of patients in the age subgroups it is impossible to perform statistical analysis of gender - age characteristics of liver stiffness in children with hepatitis. Then sensitivity and specificity of diagnostic tests and threshold value of stiffness in patients with hepatitis were determined.

ROC-analysis of Young modulus value was done. This analysis enabled to determine the threshold value of interval variable of a predictor. The more convex is the ROC curve, the more accurate are the prognostic test results. The indicator of this feature is the area under the ROC curve (AUC) which equals 0.5 for zero degree of prognosis and for maximal prognosis it equals 1 .

ROC analysis results are presented in Figure 4 and Table 2. In our study while building the ROC curve of Young modulus the area was 0.934 (corresponding to a good model of classifier), the confidence interval was 0.874-0.994;

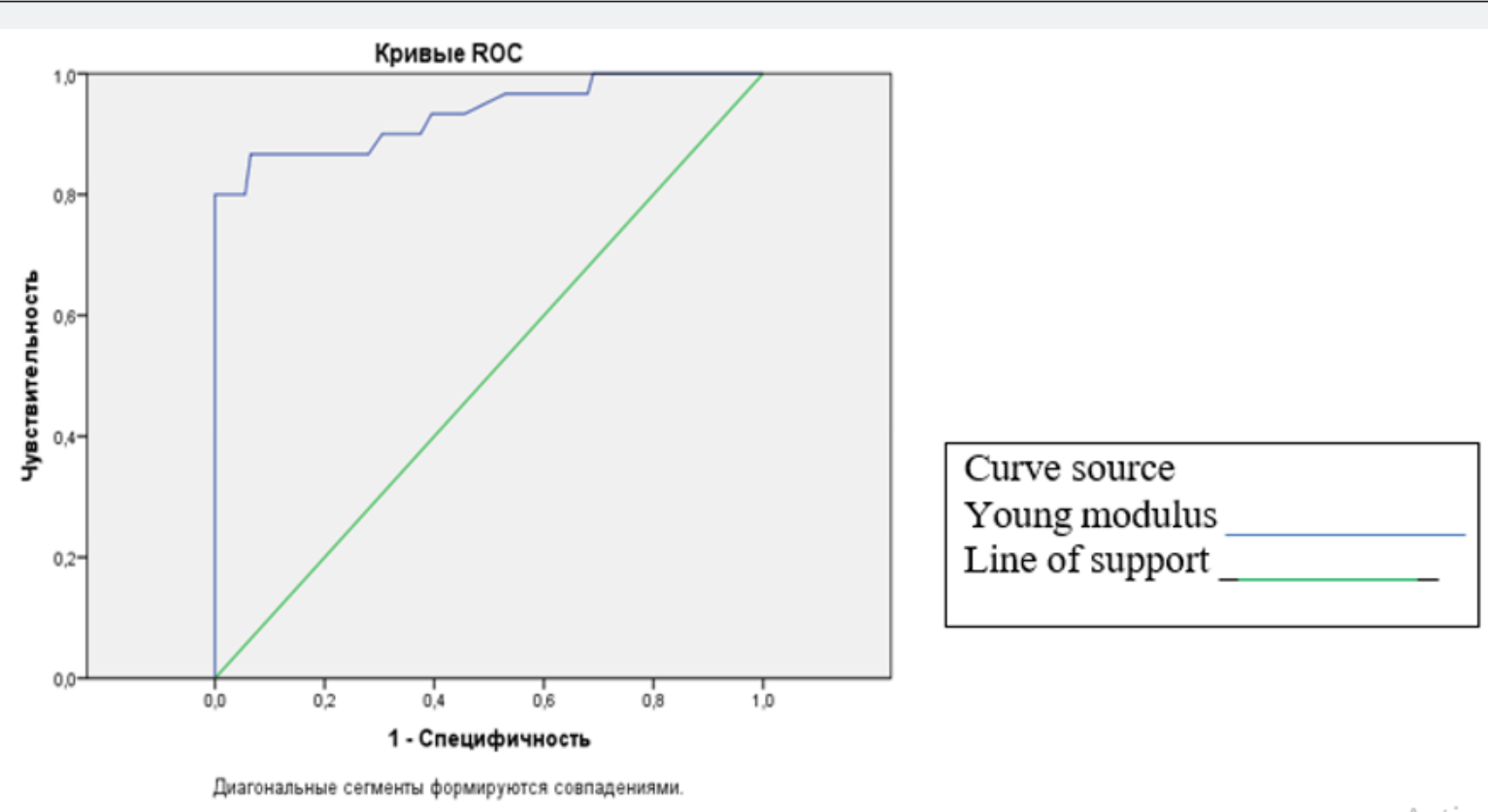

Figure 4: ROC curve of sensitivity and specificity of Young modulus value in patients with hepatitis.

Table 2: Area below ROC-curve, test variable - stiffness, Young modulus.

\begin{tabular}{|c|c|c|c|c|}
\hline Area & $\begin{array}{c}\text { Standard } \\
\text { Error }\end{array}$ & $\begin{array}{c}\text { Asymptomatic } \\
\text { Value }\end{array}$ & \multicolumn{2}{|c|}{$\begin{array}{c}\text { Asymptomatic 95\% } \\
\text { Confidence Interval }\end{array}$} \\
\hline \multirow{2}{*}{0.934} & 0.031 & 0 & Upper bound & Lower bound \\
\cline { 3 - 5 } & & 0.874 & 0.994 \\
\hline
\end{tabular}

a) Sensitivity.

b) Specificity.

c) Diagonal segments are formed by coincidences.
Table 3: Sensitivity and specificity of Young modulus values in patients with hepatitis.

\begin{tabular}{|c|c|c|}
\hline $\begin{array}{c}\text { Threshold Value of Young } \\
\text { Modulus kPa }\end{array}$ & Sensitivity, \% & Specificity, \% \\
\hline 5.71 & 80 & 95 \\
\hline 5.9 & 80 & 96 \\
\hline 6.35 & 80 & 100 \\
\hline
\end{tabular}

Table 3 shows data of sensitivity and specificity of threshold values of Young modulus values in patients with hepatitis in 
optimal combination of sensitivity and specificity predicative significance of threshold value of 5.9-6.35kPa seems optimal.

\section{Discussion}

According to literature data several research teams studied liver stiffness values in patients with hepatitis. Young modulus values in adult patients with hepatitis $\mathrm{C}$ were studied by $\mathrm{G}$ Ferraioli et al. [13]. The study group included 121 patients, accuracy of two-dimensional shear wave elastography (SWE) compared to transient elastography (TE) was evaluated, liver biopsy served as a reference standard. The study was done by two doctors by convex sensor SC6-1 and probe M3 in the right liver lobe through the intercostal space with a patient in the supine position. Evaluation of reproducibility was studied simultaneously. It was established that liver stiffness values increased simultaneously with fibrosis degree when assessed on different devices. Median of Young modulus for F1 fibrosis was $6.2 \mathrm{kPa}$ (interquartile range 5.1 - 6.8), for $\mathrm{F} 2-7.6 \mathrm{kPa}(7.2-8.3)$, for F3 - 10.0kPa (9.2-10.1), for F4 - 15.6kPa (12.8-18.8). Taking into consideration histologic verification fibrosis evaluation accuracy by SWE technique was $83.1 \%$, while by TE technique $66.7 \%$. Diagnostic accuracy for fibrosis differentiation of F0-F1 from F2-F4 with AUC was 0.92 (CI 95\%, 0.85-0.96) for SWE and 0.84 (CI95\% 0.76-0.90) for TE $(\mathrm{p}=0.002)$; for differentiation of F0-F2 from F3-F4 - 0.98 (CI 95\% 0.94-1.00) for SWE and 0.96 (CI 95\% 0.90-0.99) for TE $(\mathrm{p}=0.14)$ for differentiation $\mathrm{F} 0-\mathrm{F} 3$ from F4 - 0.98 (CI 95\% 0.93-1.00) for SWE and 0.96 (CI 95\% 0.91$0.99)$ for $T E(p=048)$. The study results showed that SWE in the real-time mode is more accurate than TE to evaluate significant fibrosis ( $>$ F2). Evaluating the results consistency obtained by the first and second research teams the value range of correlation coefficient was 0.95 (CI 0.93-0.98) and 0.93 (CI 0.90-0.96) for the first operator and the second operator correspondingly. Inter - observational consistency was 0.88 (CI 95\% 0.82-0.94) which proved the technique to have good reproducibility when it is used both by one and different operators [13].

Similar study design was carried out by a research team in hepatology of A Guibal et al [14]. Diagnostic capacity of twodimensional shear wave elastography was compared to transient elastography, histomorphology analysis served as a reference standard. The study showed high accuracy of two-dimensional shear wave elastography in evaluating liver fibrosis in 149 adult patients with chronic liver disease. Five measurements in liver segment $\mathrm{V}$ with patient in a supine position, holding breath for 5 sec and using convex sensor SC6-1 were made. Median of five successful measurements was recorded, when the light box was filled by $2 / 3$ and stiffness values were higher than $0.2 \mathrm{kPa}$. In the subgroup of random patients five additional measurements of stiffness were also made by the third physician to study the inter - observational reproducibility. All patients had liver biopsy in segment $\mathrm{V}$ under ultrasound control, the results were evaluated in accordance with METAVIR system. In the subgroup of 55 patient's stiffness correlation measured by SWE and TE and fibrosis degree were compared by means of Spirmen ranging coefficient.

The average liver stiffness in the study group was $7.0 \mathrm{kPa}$ (IQR: 6.0 ; 8.3) for F0-F1; 9.5kPa (IQR: 7.8; 11.4) for F2; $13.0 \mathrm{kPa}$ (IQR: $10.4 ; 16.7$ for F3 and 25.8kPa (IQR: $21.7 ; 34.5$ ) for F4. Twenty-five patients were included in a subgroup to study reproducibility. ICC composed 0.92 (CI 95\% 0.81-0.96). Young modulus threshold value for fibrosis $\mathrm{F}>2$ was established as $8.8 \mathrm{kPa}$ for SWE and 7.7kPa for TE with sensitivity 90.5 (69.998.8) and 85.7 (63.7-97.0) and specificity 79.4 (62.1-91.3) and 88.2 (72.5-96.7) correspondingly. For $\mathrm{F}>3$ it was established as 11.5kPa for SWE and $8.6 \mathrm{kPa}$ for TE with sensitivity 78.6 (49.295.3) and 85.7 (57.2-98.2), specificity 97.6 (87.1-99.9) and 90.2 (76.9-97.3) correspondingly. For fibrosis $\mathrm{F}=4$ it was established as $15.8 \mathrm{kPa}$ for SWE and $11.8 \mathrm{kPa}$ for TE with sensitivity 100 (54.1-100) and 100 (54.1-100), specificity 98.0 (89.1-99.9) and 87.8 (75.2-95.4) correspondingly. Two-dimensional shear wave elastography showed high diagnostic accuracy for fibrosis F >2 with AUC 0.904 (CI 95\% 0.845-0.946); 0.958 (CI 95\% 0.912-0.984) for fibrosis $\mathrm{F}>3$ and 0.988 (CI 95\% 0.955-0.999 for fibrosis $=\mathrm{F} 4$. There was established significant correlation between fibrosis stage and stiffness by SWE ( $\mathrm{r}=0.77$; CI 95\% 0.63-0.86; $\mathrm{p}<0.0001)$ and TE $(\mathrm{r}=0.65$; CI 95\% 0.47-0.78; $\mathrm{p}<0.01$ ) in this subgroup [14].

Two hundred and twenty-six adult patients with hepatitis B included in V Y Leung et al. [15] underwent liver biopsy. The stiffness values were compared to those in 171 healthy volunteers from the control group. Measurements were made by a convex sensor in segment VIII of the right liver lobe by three operators with different work experience, patients holding breath for a short time. Reproducibility and degree of values consistency were evaluated. Inconsistency of elastometry results and histologic evaluation was $10.2 \%$ (23 of 226 patients). Threshold value for fibrosis > F1 was determined as 6.5kPa with AUC 0.86; for $>$ F2 as $7.1 \mathrm{kPa}$ with AUC 0.88; for $>\mathrm{F} 3$ as $7.9 \mathrm{kPa}$ with AUC 0.93; for F4 as $10.1 \mathrm{kPa}$ with AUC 0.98 . The range of values of correlation coefficient for three operators measuring stiffness in three different hepatic areas was from 0.86 to 0.98 , CI $95 \%$ 0.71 to 0.99 . Good reproducibility was noted among the three operators (ICC 0.85; confidence interval 95\% 0.70, 0.94 [15]. Comparison of results of shear wave elastography and transient elastography in the diagnosis of diffuse liver diseases was carried out in the group of 128 patients in the study performed by Diomidova VN \& Petrova OV [16]. Liver stiffness was studied in 60 practically healthy people and 68 patients with chronic liver diseases. Stiffness values (Emean) in segment VIII projection were significantly higher $(\mathrm{p}<0.05)$ when comparing with other segments. In transient elastography stiffness values in patients with chronic viral hepatitis $\mathrm{B}$ and $\mathrm{C}$ were $7.2 \mathrm{kPa}$, liver cirrhosis - 43.8kPa; in shear wave elastometry (Emean) - 8.3 and $55.3 \mathrm{kPa}$ correspondingly ( $\mathrm{p}<0.05$ comparing to the control group). 
Efficiency rate of measurements by transient elastography was $84.4 \%$ of cases, shear wave elastometry $-100.0 \%$ [16].

It is known from the literature data that $\mathrm{O}$ Belei et al. research team [11] carried out the study of the feasibility of liver stiffness measurement in children with CLD by means of point shear wave elastography (ARFI) and two-dimensional elastography (SWE) compared to transient elastography (TE) as a reference standard. Liver biopsy in children was not performed. The group of children with liver diseases (n 54) included: children with chronic hepatitis $\mathrm{B}$, unspecified chronic hepatitis, autoimmune hepatitis, nonalcoholic steatohepatitis, Wilson disease and hemochromatosis. Ten liver stiffness measurements were made. SWE was made by Aixplorer device (Supersonic Imagine, Aixen-Provence, France) using a convex sensor, a mean value of 5 measurements was determined. The mean value of Young modulus in the children studied by two- dimensional shear wave elastography SWE was (7.76 \pm 2.46$) \mathrm{kPa}$ (CI 95\% 7.07-8.46), which is sufficiently close to our study.

Using the technique of two-dimensional shear wave elastography (Aixplorer, Supersonic Imagine, France) in patients with chronic liver diseases (76 children) 0 Tutar et al. [12] also established that the mean value of liver stiffness in children with fibrosis changes of F1, F2, F3 and F4 degrees according to Brunt was significantly higher than in the control group $(p<0.001$ for all comparisons) [12].

The analysis of the data obtained in our study as well as that of other research teams has revealed that values of hepatic tissue stiffness in patients with chronic liver diseases are significantly higher than those in healthy volunteers. Young modulus values in the study group are significantly higher than those of the children in the control group (Emean median - 7.05 and $5.00 \mathrm{kPa}$, interquartile range $-6.40-9.18$ and 4.70 $5.38 \mathrm{kPa}$ correspondingly) ( $\mathrm{p}<0.001$ ). In children with chronic liver diseases the mean value of Young modulus of $7.89 \pm$ $0.43 \mathrm{kPa}$ coincides with the data of 0 Belei et al. [11] research team $-7.76 \pm 2.46 \mathrm{kPa}$ and close to the results of A Guibal et al. [14] - 7.0kPa. Young modulus value of $5.9-6.35 \mathrm{kPa}$ used as a test (close to the results of V Y Leung et al. [15] study) will enable to reveal children who need additional $80 \%$ sensitivity and $95 \%$ specificity examination. Taking into consideration the noninvasive feature of this technique ultrasound elastometry can be used both in complex evaluation of liver lesions and for dynamic monitoring purposes.

\section{References}

1. Orlovsky DV, Orlovsk, N, Yu Oshmyanskaya, Nedzvetskaya NV (2013) Place of puncture biopsy in diagnosis of chronic diffuse liver diseases. Gastroenterology 48(2): 47-52.

2. Uchaikin VF, Chuyelov SB, Rossina AL, et al. (2008) Cirrhosis of the liver in children. Pediatrics 87(5): 52-57.

3. Zaprudnov AM (2016) Pediatric gastroenterology: formation, development, study prospects. The Russian herald of perinatology and pediatrics 1: 7-14.

4. Rockey DC, Caldwell SH, Goodman ZD, Nelson RC, Smith AD, et al. (2009) Liver biopsy. Hepatology 49(3): 1017-1044.

5. Czaja AJ, Carpenter HA (2007) Optimizing diagnosis from the medical liver biopsy. Clin Gastroenterol Hepatol 5(8): 898-907.

6. Dezsofi A, Baumann U, Dhawan A, Durmaz O, Fischler B, et al. (2015) Liver Biopsy in Children: Position Paper of the ESPGHAN Hepatology Committee. J Pediatr Gastroenterol Nutr 60(3): 408-420.

7. Ovchinsky NR, Moreira K, Lefkowitch JH, Lavine JE (2012) The Liver Biopsy in Modern Clinical Practice: A pediatric Point-of-View. Adv Anat Pathol 19(4): 250-262.

8. Mitkov VV, Mitkova MD (2015) Ultrasound shear wave elastography. Ultrasound and Functional Diagnostics 2: 94-108.

9. Pykov MI, Kuzmina NE, Yu Kinzersky A (2017) Study of normal liver stiffness parameters in children using the Ultrasound Shear Wave Elastography. Pediatria Named after G.N. Speransky 4: 63-69.

10. Franchi-Abella S, Corno L, Gonzales E, Antoni G, Fabre M, et al. (2016) Feasibility and diagnostic accuracy of supersonic shear-wave elastography for the assessment of liver stiffness and liver fibrosis in children: a pilot study of 96 patients. Radiology 278(2): 554-562.

11. Belei O, Sporea I, Gradinaru-Tascau O, Olariu L, Popescu A, et al. (2016) Comparison of three ultrasound based elastographic techniques in children and adolescents with chronic diffuse liver diseases. Med Ultrason 18(2): 145-150.

12. Tutar, O, Beser OF, Adaletli I, Tunc N, Gulcu D, et al. (2014) Shear wave elastography in the evaluation of liver fibrosis in children. J Pediatr Gastroenterol Nutr 58(6): 750-755.

13. Ferraioli G, Tinelli C, Dal Bello B, Zicchetti M, Filice G, et al. (2012) Accuracy of real-time shear wave elastography for assessing liver fibrosis in chronic hepatitis C: a pilot study. Hepatology 56(6): 21252133.

14. Guibal A, Renosi G, Rode A, Scoazec JY, Guillaud O, et al. (2016) Shear wave elastography: An accurate technique to stage liver fibrosis in chronic liver diseases. Diagn Interv Imaging 97(1): 91-99.

15. Leung VY, Shen J, Wong VW, Abrigo J, Wong GL, et al. (2013) Quantitative elastography of liver fibrosis and spleen stiffness in chronic hepatitis B carriers: comparison of shear-wave elastography and transient elastography with liver biopsy correlation. Radiology 269(3): 910-918.

16. Diomidova VN, Petrova OV (2013) Comparative Analysis of Shear Wave Elastography and Transient Elastography in Diagnosis of Diffuse Liver Disease. Ultrasound and Functional Diagnostics 5: 17-23. 


\section{Your next submission with Juniper Publishers} will reach you the below assets

- Quality Editorial service

- Swift Peer Review

- Reprints availability

- E-prints Service

- Manuscript Podcast for convenient understanding

- Global attainment for your research

- Manuscript accessibility in different formats

( Pdf, E-pub, Full Text, Audio)

- Unceasing customer service

Track the below URL for one-step submission https://juniperpublishers.com/online-submission.php 